\title{
Increased risk for urological cancer associated with anxiety disorder: a retrospective cohort study
}

\author{
Yung-Chan Chen ${ }^{1}$, Li-Ting Kao ${ }^{2}$, Herng-Ching Lin ${ }^{3}$, Hsin-Chien Lee ${ }^{1,3,4}$, Chung-Chien Huang ${ }^{5+}$ \\ and Shiu-Dong Chung $3,6,7,8^{*}+$
}

\begin{abstract}
Background: Anxiety disorders (ADs) are common with a high rate of medical comorbidities. Although the association between ADs and the overall cancer risk remains controversial, patients with ADs were found to be more likely to develop specific cancer types. Herein, we estimated the risk of developing urological cancers among patients with ADs in a 5-year follow-up period using a population-based database.

Methods: Two study cohorts were identified from the Taiwan Longitudinal Health Insurance Database 2005: patients with ADs, and comparison subjects selected by one-to-one matching for sex, age, and the year of recruitment. Follow-up was undertaken to determine whether sampled patients and comparison subjects had developed urological cancers in the subsequent 5 years.

Results: We found that urological cancers occurred among $0.54 \%$ of patients with ADs and $0.13 \%$ of comparison subjects. After adjusting for sociodemographic characteristics, medical comorbidities, and alcohol and tobacco use disorder, the stratified Cox proportional hazard regression suggested that patients with ADs were more likely to develop urological cancers relative to comparison subjects (adjusted hazard ratio, 3.67; 95\% confidence interval, 2. $85 \sim 4.72$ ). The adjusted HR for males with ADs was 3.82 ( $95 \%$ Cl: $2.79 \sim 5.23$ ) in comparison to males without ADs. In addition, the adjusted HR for females with ADs was 3.47 (95\% Cl: $2.26 ~ 5.31$ ) than those females without ADs.
\end{abstract}

Conclusions: We concluded that during the 5-year follow-up period, there was a significantly increased risk of urological cancers among patients with ADs.

Keyword: Anxiety disorder, Urological cancer, Bladder cancer, Epidemiology

\section{Background}

Anxiety disorders (ADs), including panic disorder, generalized anxiety disorder, phobic disorder and so on, are common mental disorders within a disease category characterized by excessive worry which might affect $9.2 \% \sim 28.7 \%$ of the general population around the world $[1,2]$. The prevalence of ADs is not only high but also strikingly increasing in Asian populations [3, 4].

ADs are shown to markedly compromise the quality of life and psychosocial functioning in several domains [5].

\footnotetext{
* Correspondence: chungshiudong@gmail.com

${ }^{\dagger}$ Equal contributors

${ }^{3}$ Sleep Research Center, Taipei Medical University, Taipei, Taiwan

${ }^{6}$ Division of Urology, Department of Surgery, Far Eastern Memorial Hospital,

New Taipei City, Taiwan

Full list of author information is available at the end of the article
}

ADs are highly comorbid with numerous chronic medical illness including diabetes, coronary artery disease, congestive heart failure, asthma, chronic obstructive pulmonary disease, and arthritis [6, 7]. Thus, the research focus has gradually turned to the relationship between $\mathrm{ADs}$ and malignancies. Indeed, psychological distress was proven to have an adverse effect on cancer survival $[8,9]$.

Nevertheless, it is still controversial and unclear whether the existence of ADs is associated with the subsequent occurrence of cancer [8, 10-13]. Patients with ADs were found to be at increased risks of developing specific cancer types including lung, brain, and prostate cancers [11-13]. Although the underlying mechanism remains obscure, indirect relationships through a 
surveillance bias and unhealthy lifestyle behaviors were proposed to interpret this association [12, 13]. It is debatable whether these factors could contribute to the overall cancer risk instead of to certain cancer types such as prostate and other urological cancers found in previous studies.

It is possible that the weak, though significant, association seen in previous studies lends support to a direct relationship between ADs and urological cancers. Even so, there is evidence that dysfunction of the serotonin receptor pathway, crucial in anxiety disorders, might also be involved in the proliferation of urological cancer $[14,15]$. This could pose a threat to both a patient's mental and physical health. Considering the biological plausibility, this study aimed to investigate the relationship between ADs and the subsequent occurrence of urological cancers using a longitudinal population-based database.

\section{Methods}

\section{Database}

The data for this retrospective cohort study were sourced from the Taiwan Longitudinal Health Insurance Database 2005 (LHID2005). The LHID2005, compiled by the Taiwan National Health Research Institute, consists of original medical claims data for 1,000,000 individuals randomly sampled from all enrollees $(n=25.68$ million) in the Taiwan National Health Insurance (NHI) program in 2005. Since around $96 \%$ of the Taiwanese population enrolled in the NHI program, the LHID2005 provides an exclusive opportunity for researchers to follow-up the use of all medical services for nationwide, population-based sampled subjects. Hundreds of studies utilizing this dataset have been published in internationally peer-reviewed journals [16].

This study was exempt from full review by the Institutional Review Board of National Defense Medical Center since the LHID2005 consists of de-identified secondary data released to the public for research purposes.

\section{Study sample}

This study features a study cohort and a comparison cohort. The study cohort included all patients who had received one of the following $\mathrm{ADs}$ diagnoses between January 1, 2001 and December 31, 2008: panic disorder (ICD-9-CM: 300.01), generalized anxiety disorder (ICD9-CM: 300.02), phobic disorder (ICD-9-CM: 300.2, $300.20 \sim 300.29$ ), obsessive-compulsive disorder (ICD-9CM: 300.3$)$ and acute stress reaction \& post-traumatic stress disorder (ICD-9-CM: 308, 309.81 309.83) ( $n=$ $143,329)$. In order to increase the diagnosis validity, this study only included patients with ADs who received at least two anxiety disorder diagnoses with at least one diagnosis having been made by a board-certified psychiatrist $(n=71,065)$. We assigned the date of the second medical service utilization for ADs during the recruitment period as the index date. We then excluded patients aged under 18 years $(n=1,961)$ in order to limit the study to the adult population. Additionally, subjects who had a history of major psychiatric disorders or a substance-related disorder (ICD-9-CM codes $290 \sim 299$ or $303 \sim 305) \quad(n=3,236)$ were excluded in order to exclude possible confounding effects of other mental illnesses on the association between ADs and urological cancers. We further excluded those subjects who were diagnosed with any type of cancer (ICD-9-CM codes $140 \sim 239)$ prior to the index date $(n=7,265)$. As a result, 58,603 subjects with ADs were included in the study cohort. The selection procedures were shown in Fig. 1.

We likewise extracted the comparison cohort from the LHID2005. We randomly selected one comparison subject for every study subject, matched by sex, age (18 $30,30 \sim 39,40 \sim 49,50 \sim 59,60 \sim 69$, and $>69$ years), and index year. While for the study cohort, the year of the index date was the year in which the study subjects received their first diagnosis of ADs, for the comparison

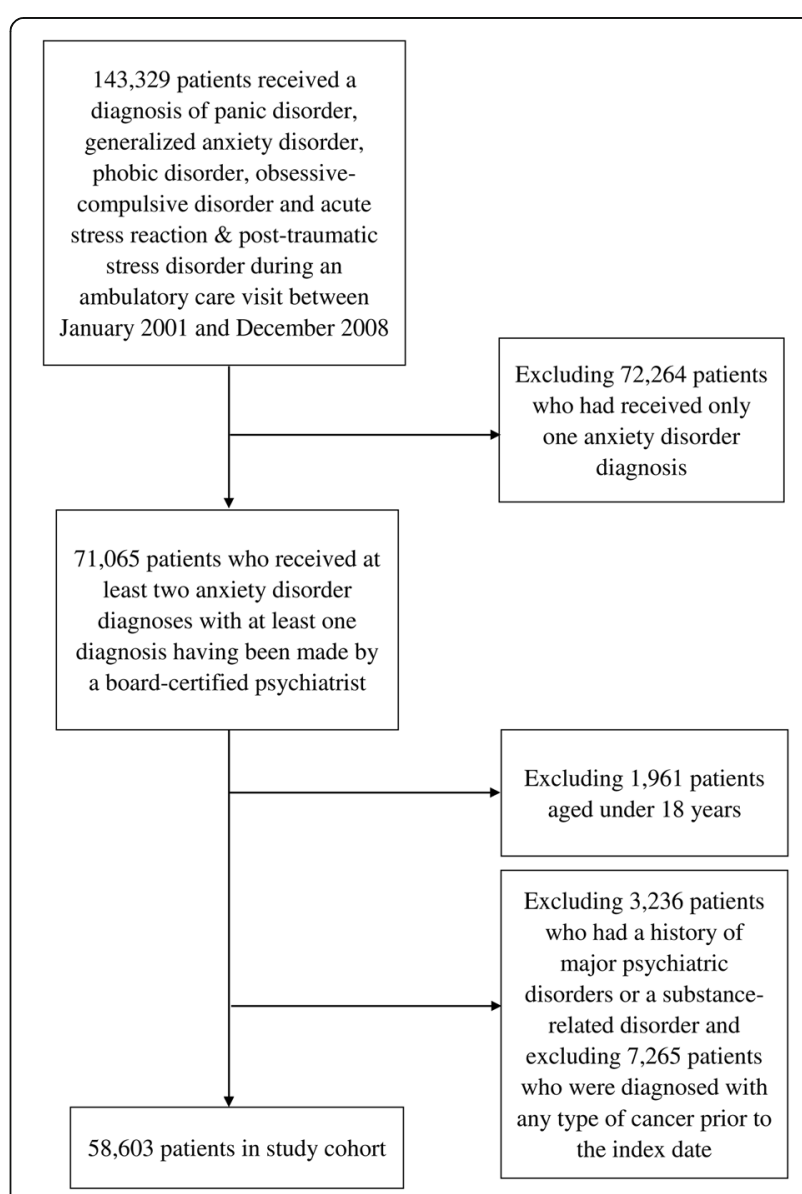

Fig. 1 Flow diagram for study cohort 
cohort, the year of the index date was simply a matched year in which comparison subjects had a medical utilization. We assigned the date of their first use of medical services occurring during that matched year as the index date for the comparison cohort. We ensured that no selected comparison subjects had ever received a diagnosis of ADs since initiation of the Taiwan NHI program in 1995. We also assured that no selected comparison subjects had ever received a diagnosis of major psychiatric disorders, substance-related disorder, or any type of cancer prior to the index date.

Thereafter, each patient in the study $(n=117,206)$ was individually tracked for a 5 -year period from their index date to identify whether or not they had received a diagnosis of urological cancer (ICD-9-CM code185 189). The diagnoses of urological cancers have been confirmed by the registry of catastrophic illness. For the occurrence of cancer to be reported in the registry, histological confirmation is required.

\section{Statistical analysis}

All statistical analyses were performed by the SAS statistical package (SAS System for Windows, vers. 8.2, Cary, NC, USA). We used the Kaplan-Meier method to estimate the 5-year cancer-free survival rate and the log-rank test to examine differences in the risks of urological cancer xbetween these two cohorts. We used stratified Cox proportional hazard regressions, stratified by sex, age group, and index year to calculate the cancer hazard for the study cohort relative to the comparison cohort. We adjusted for demographic and socioeconomic characteristics, medical comorbidities, and alcohol and tobacco use in the regression model. The variables of socioeconomic characteristics included monthly income (New Taiwan (NT) $\$ 0 \sim 15,840$, NT $\$ 15,841 \sim 25,000, \geq \mathrm{NT} \$ 25,001$; the average exchange rate in 2011 was US\$1 $\approx$ NT\$29), geographical location (northern, central, eastern, and southern Taiwan), and urbanization level of each subject's residence (5 levels, with 1 indicating the most urbanized and 5 the least urbanized). NT $\$ 15,840$ was used as the first cut-off value as it is the government-stipulated minimum wage for full-time employees in Taiwan. Medical comorbidities selected in this study were hypertension, diabetes, obesity, alcohol abuse, and tobacco use disorder. All medical comorbidities were defined by using ICD-9-CM codes in LHID2005. In addition, we censored subjects who died from non-cancer causes during the 5-year follow-up period in the regression model. Of the sampled subjects, 3749 died from non-cancer causes, including 2051 from the study cohort (3.5\% of study cohort) and 1698 from the comparison cohort (2.9\% of the comparison cohort). The differences were considered significant with a two-sided $p$ value of $\leq 0.05$.

\section{Results}

Of the 117,206 study subjects, the mean age was 51.0 years (standard deviation, 16.5 years), while they were 51.1 years for the study cohort and 50.9 years for the comparison cohort $(p=0.209)$. Table 1 shows the demographic and socioeconomic characteristics of subjects with and without ADs. The overwhelming majority (61.8\%) of study subjects were female, and over half were aged $<50$ years (about 57\%). There were significant differences between the study and comparison cohorts in terms of monthly

Table 1 Subjects with anxiety disorder and a comparison group in relation to sociodemographic characteristics and medical comorbidities in Taiwan $(n=117,206)$

\begin{tabular}{|c|c|c|c|c|c|}
\hline \multirow[t]{2}{*}{ Variable } & \multicolumn{2}{|c|}{$\begin{array}{l}\text { Patients with } \\
\text { anxiety disorder } \\
n=58,603\end{array}$} & \multicolumn{2}{|c|}{$\begin{array}{l}\text { Comparison cohort } \\
n=58,603\end{array}$} & \multirow[t]{2}{*}{$p$ value } \\
\hline & No. & Percent & No. & Percent & \\
\hline Sex & & & & & 1.000 \\
\hline Male & 22,394 & 38.2 & 22,394 & 38.2 & \\
\hline Female & 36,209 & 61.8 & 36,209 & 61.8 & \\
\hline Age (years) & & & & & 1.000 \\
\hline$<40$ & 19,722 & 33.8 & 19,722 & 33.8 & \\
\hline $40 \sim 49$ & 13,269 & 22.7 & 13,269 & 22.7 & \\
\hline $50 \sim 59$ & 10,654 & 18.2 & 10,654 & 18.2 & \\
\hline $60 \sim 69$ & 7,405 & 12.7 & 7,405 & 12.7 & \\
\hline$>69$ & 7,323 & 12.6 & 7,323 & 12.6 & \\
\hline Monthly income & & & & & $<0.001$ \\
\hline$\leq \mathrm{NT} \$ 15,840$ & 24,159 & 41.2 & 23,299 & 39.8 & \\
\hline NT\$15,841 25,000 & 22,503 & 38.4 & 22,108 & 37.7 & \\
\hline$\geq \mathrm{NT} \$ 25,001$ & 11,941 & 20.4 & 13,196 & 22.5 & \\
\hline Geographic region & & & & & $<0.001$ \\
\hline Northern & 25,542 & 43.6 & 27,622 & 47.1 & \\
\hline Central & 16,292 & 27.8 & 13,642 & 23.3 & \\
\hline Southern & 15,574 & 26.6 & 15,797 & 27.0 & \\
\hline Eastern & 1,195 & 2.0 & 1,542 & 2.6 & \\
\hline Urbanization level & & & & & $<0.001$ \\
\hline 1 (most urbanized) & 16,858 & 28.8 & 17,685 & 30.2 & \\
\hline 2 & 16,235 & 27.7 & 16,224 & 27.7 & \\
\hline 3 & 8,593 & 14.7 & 9,509 & 16.2 & \\
\hline 4 & 9,217 & 15.7 & 8,288 & 14.1 & \\
\hline 5 (least urbanized) & 7,700 & 13.1 & 6,897 & 11.8 & \\
\hline Obesity & 670 & 1.1 & 390 & 0.7 & $<0.001$ \\
\hline $\begin{array}{l}\text { Alcohol abuse/ } \\
\text { alcohol dependence } \\
\text { syndrome }\end{array}$ & 790 & 1.4 & 69 & 0.1 & $<0.001$ \\
\hline Tobacco use disorder & 1,497 & 2.6 & 355 & 0.6 & $<0.001$ \\
\hline Diabetes & 6,611 & 11.3 & 5,483 & 9.4 & $<0.001$ \\
\hline Hypertension & 18,871 & 32.2 & 12,041 & 20.6 & $<0.001$ \\
\hline
\end{tabular}

Note: In 2011, the average exchange rate was US\$1 $\approx$ New Taiwan (NT) \$29 
income, geographical location, and urbanization level of each subject's residence. In addition, subjects with ADs had a higher prevalence of medical comorbidities of hypertension, diabetes, obesity, and alcohol and tobacco use problems than subjects without $\mathrm{ADs}$ (all $p<0.001$ ).

Table 2 presents the incidence rate for a new urological cancer diagnosis by cohort within the 5-year follow-up period. Of the 117,206 subjects, 391 (0.33\%) received a diagnosis of urological cancer: 315 in the study cohort $(0.54 \%$ of the subjects with anxiety disorder) and 76 in the comparison cohort $(0.13 \%$ of subjects without anxiety disorder). The incidence rates of urological cancers during the 5-year follow-up period were $10.93(9.76 \sim 12.21)$ per 10,000 person-years for the study cohort and $2.62(2.07 \sim 3.28)$ per 10,000 person-years for the comparison cohort. The log-rank test revealed that subjects with ADs had a significantly lower 5-year urological cancer-free survival rate than comparison subjects $(p=0.005)$. Figure 2 presents results of the Kaplan-Meier survival analysis.

Results of the stratified Cox proportional regressions (stratified on sex, age group, and index year) are also presented in Table 2. The crude hazard ratio (HR) for urological cancer during the 5-year follow-up period for the study cohort was 4.16 (95\% confidence interval (CI): $3.23 \sim 5.34$ ) with respect to the comparison cohort. After adjusting for urbanization level, monthly income, geographic region, hypertension, diabetes, obesity, and alcohol and tobacco use, and censoring cases that died from non-cancer causes, the HR for urological cancer during the 5-year follow-up period for study cohort was 3.67 (95\% CI: $2.85 \sim 4.72$ ) that of comparison patients.

Table 3 presents the proportional HRs of subgroups classified by the type of urological cancers (kidney cancer, bladder cancer, prostate cancer, and others). The adjusted HRs for kidney cancer, bladder cancer, prostate cancer, and others for subjects with ADs were 2.84 (95\% CI: $1.73 \sim 4.66$ ), 2.94 (95\% CI: $1.89 \sim 4.58$ ), 4.67 (95\% CI: $3.06 \sim 7.12$ ), and 6.75 (95\% CI: $2.01 \sim$ 22.69), respectively, relative to those without ADs.

The incidences of urological cancers within 5 years after the index date between patients with $\mathrm{ADs}$ and those without ADs stratified by sex are presented in Table 4 . The adjusted HR for males with ADs was 3.82 (95\% CI: $2.79 \sim 5.23)$ in comparison to males without ADs. In addition, the adjusted HR for females with ADs was 3.47 (95\% CI: $2.26 \sim 5.31$ ) than those females without ADs.

\section{Discussion}

A relationship exists between ADs and the subsequent occurrence of urological cancers, as suggested by the results herein. The findings of this study were consistent with previous reports $[12,13]$. Shen and colleagues reported that male patients with generalized ADs had an increased standardized incidence ratio (SIR) for prostate cancer $(2.17,95 \%$ CI: $1.56 \sim 2.93)$ [13]. Since the prevalence of ADs in the general population is substantial, the observed SIR may introduce bias and cause underestimation of the true relative risk [17]. Another study by Liang and colleagues only demonstrated an increased risk of developing prostate cancer among patients with ADs older than 65 years [12]. The failure to exclude comorbid psychiatric disorders made the study group more heterogeneous which could have biased the results toward zero.

Both studies argued that the higher risk of prostate cancer could be partially explained by a surveillance bias. Patients with ADs were likely to search for medical help and thus might have received more imaging and laboratory examinations $[6,7,18]$. If true, the overall risk of cancer should also increase among patients with ADs, which was not supported by previous study results. Patients with ADs have more urological complaints $[19,20]$. Liang and colleagues further validated their assumption regarding a surveillance bias by showing a higher prostate-specific antigen (PSA) screening rate among patients with ADs [12]. However, the proposed explanation is difficult to apply to the significantly increased risks of urological cancers other than prostate cancer among patients with ADs.

Another explanation stems from unhealthy lifestyles and medication use among patients with ADs. Patients with ADs tend to be overweight and physically inactive and are more likely to use alcohol and tobacco [21, 22].

Table 2 Hazard ratios (HRs) and 95\% confidence intervals (Cls) of urological cancer among sampled subjects during the 5-year follow-up period from the index date

\begin{tabular}{|c|c|c|c|}
\hline \multirow[t]{2}{*}{ Presence of urological cancer } & Total sample $n=117,206$ & Patients with anxiety disorder $n=58,603$ & Comparison cohort $n=58,603$ \\
\hline & Percent & Percent & Percent \\
\hline Yes & $391(0.33)$ & $315(0.54)$ & $76(0.13)$ \\
\hline Incidence rate per 10,000 person-years ( $95 \% \mathrm{Cl}$ ) & $6.76(6.11 \sim 7.47)$ & $10.93(9.76 \sim 12.21)$ & $2.62(2.07 \sim 3.28)$ \\
\hline Crude HR ${ }^{a}(95 \% \mathrm{Cl})$ & - & $4.16^{* * *}(3.23 \sim 5.34)$ & 1.00 \\
\hline Adjusted HR $(95 \% \mathrm{Cl})$ & - & $3.67^{* * *}(2.85 \sim 4.72)$ & 1.00 \\
\hline
\end{tabular}

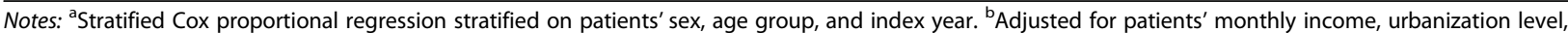
geographic region, hypertension, diabetes, obesity, tobacco use disorder, and alcohol abuse/alcohol dependence syndrome ${ }^{* * *} p<0.001$ 


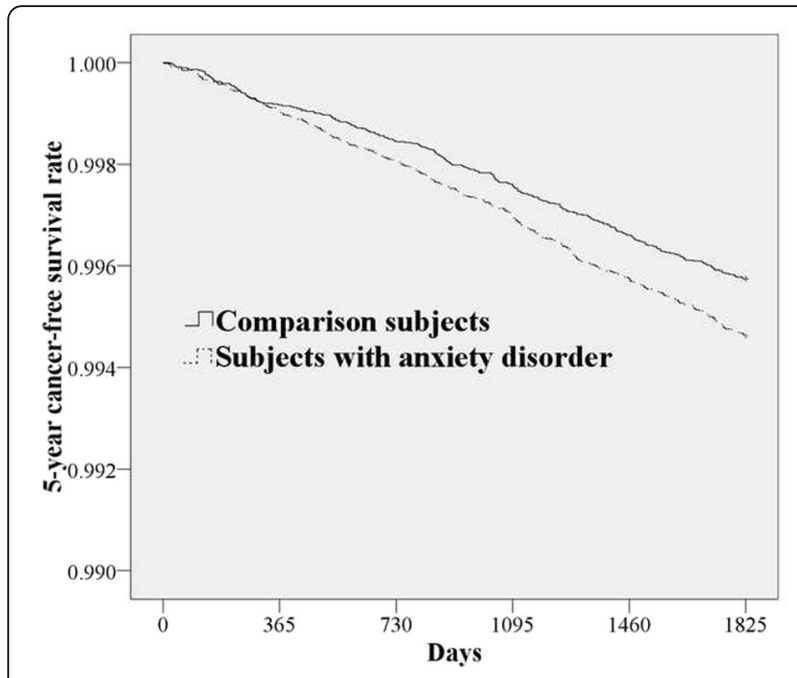

Fig. 2 Urological cancer-free survival rates for subjects with anxiety disorder and comparison subjects

In addition, a stress-prone personality and unfavorable coping styles commonly seen among patients with ADs might place them at a higher cancer risk [8]. Even so, the overall cancer risks were not consistently high in previous studies $[11,12]$.

In an earlier comprehensive review of the relationship between lifestyle issues and urological cancers, only strong connections of tobacco use with bladder and renal cancers were repeatedly supported by the literature [23]. Other lifestyle risk factors, if they existed, had only modest impacts on the development of urological malignancies. Furthermore, patients with ADs remained at a significantly high risk of developing all kinds of urological cancers after adjusting for tobacco use disorder in this study.

The long-term use of psychotropic medications, particularly benzodiazepine anxiolytics, is prevalent among patients with ADs. However, study results of the carcinogenic potential of benzodiazepine anxiolytics remain equivocal $[24,25]$. Kao and colleagues reported that patients with a history of taking benzodiazepine were more likely to develop prostate and bladder/kidney cancers with respective HRs of 1.72 and 1.76 [24]. There is still no specific mechanisms or explanations which account for the observed increased risk, and their findings were challenged for failing to control numerous confounding factors [26].

The current study demonstrated that patients with ADs were more likely to develop prostate cancer after adjusting for available demographic, socioeconomic, and comorbid medical and substance use variables. A direct mechanism linking ADs itself to urological cancers should not be neglected.

As stated before, stress-related psychosocial factors can have adverse effects on cancer incidence and survival by disturbing functions of the immune system, thus enhancing the risk of carcinogenesis [27]. Lines of evidence

Table 3 Hazard ratios (HRs) and 95\% confidence intervals (Cls) of urological cancer among sampled subjects during the 5-year follow-up period from the index date according to the type of urological cancer

\begin{tabular}{|c|c|c|c|c|}
\hline \multirow[t]{2}{*}{ Presence of urological cancer } & \multicolumn{2}{|c|}{ Patients with anxiety disorder $n=58,603$} & \multicolumn{2}{|c|}{ Comparison cohort $n=58,603$} \\
\hline & No. & Percent & No. & Percent \\
\hline \multicolumn{5}{|l|}{ Kidney cancer } \\
\hline Yes & $67(0.11)$ & & $21(0.04)$ & \\
\hline Incidence rate per 100,000 person-years $(95 \% \mathrm{Cl})$ & $23.20(17.98 \sim 29.47)$ & & $7.24(4.48 \sim 11.07)$ & \\
\hline Adjusted HR, & $2.84^{* * *}(1.73 \sim 4.66)$ & & 1.00 & \\
\hline \multicolumn{5}{|l|}{ Bladder cancer } \\
\hline Yes & $88(0.15)$ & & $26(0.04)$ & \\
\hline Incidence rate per 100,000 person-years $(95 \% \mathrm{Cl})$ & $30.48(24.45 \sim 37.55)$ & & $8.97(5.86 \sim 13.14)$ & \\
\hline Adjusted HR,,$(95 \% \mathrm{Cl})$ & $2.94^{* * *}(1.89 \sim 4.58)$ & & 1.00 & \\
\hline \multicolumn{5}{|l|}{ Prostate cancer (men only) } \\
\hline Yes & $138(0.62)$ & & $26(0.12)$ & \\
\hline Incidence rate per 10,000 person-years ( $95 \% \mathrm{Cl}$ ) & $12.53(10.53 \sim 14.81)$ & & $2.35(1.53 \sim 3.44)$ & \\
\hline Adjusted $\mathrm{HR}^{\mathrm{ab}},{ }^{\mathrm{b}}(95 \% \mathrm{Cl})$ & $4.67^{* * *}(3.06 \sim 7.12)$ & & 1.00 & \\
\hline \multicolumn{5}{|l|}{ Others } \\
\hline Yes & $22(0.04)$ & & $3(0.01)$ & \\
\hline Incidence rate per 100,000 person-years $(95 \% \mathrm{Cl})$ & $7.62(4.77 \sim 11.53)$ & & $1.04(0.21 \sim 3.02)$ & \\
\hline Adjusted HR ${ }^{\mathrm{a} b}(95 \% \mathrm{Cl})$ & $6.75^{* *}(2.01 \sim 22.69)$ & & 1.00 & \\
\hline
\end{tabular}

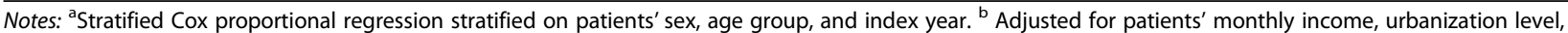
geographic region, hypertension, diabetes, obesity, tobacco use disorder, and alcohol abuse/alcohol dependence syndrome ${ }^{* *} p<0.01,{ }^{* * *} p<0.001$ 
Table 4 Hazard ratios (HRs) and 95\% confidence intervals (Cls) of urological cancer among sampled subjects during the 5-year follow-up period from the index date according to sex

\begin{tabular}{|c|c|c|c|c|c|c|c|c|}
\hline \multirow[t]{3}{*}{ Presence of urological cancer } & \multicolumn{4}{|c|}{ Males } & \multicolumn{4}{|c|}{ Females } \\
\hline & \multicolumn{2}{|c|}{$\begin{array}{l}\text { Patients with anxiety } \\
\text { disorder }(n=22,394)\end{array}$} & \multicolumn{2}{|c|}{$\begin{array}{l}\text { Comparison cohort } \\
(n=22,394)\end{array}$} & \multicolumn{2}{|c|}{$\begin{array}{l}\text { Patients with anxiety } \\
\text { disorder }(n=36,209)\end{array}$} & \multicolumn{2}{|c|}{$\begin{array}{l}\text { Comparison cohort } \\
(n=36,209)\end{array}$} \\
\hline & No. & Percent & No. & Percent & No. & Percent & No. & Percent \\
\hline Yes & \multicolumn{2}{|c|}{$209(0.93)$} & \multicolumn{2}{|c|}{$49(0.22)$} & \multicolumn{2}{|c|}{$106(0.29)$} & \multicolumn{2}{|c|}{$27(0.07)$} \\
\hline Incidence rate per 10,000 person-years $(95 \% \mathrm{Cl})$ & \multicolumn{2}{|c|}{$19.01(16.52 \sim 21.77)$} & \multicolumn{2}{|c|}{$4.43(3.27 \sim 5.85)$} & \multicolumn{2}{|c|}{$5.95(4.87 \sim 7.19)$} & \multicolumn{2}{|c|}{$1.51(0.99 \sim 2.19)$} \\
\hline Crude $\mathrm{HR}^{\mathrm{a}}(95 \% \mathrm{Cl})$ & \multicolumn{2}{|c|}{$4.28^{* * *}(3.14 \sim 5.85)$} & \multicolumn{2}{|l|}{1.00} & \multicolumn{2}{|c|}{$3.93^{* * *}(2.58 \sim 6.00)$} & \multicolumn{2}{|c|}{1.00} \\
\hline Adjusted $\mathrm{HR}^{\mathrm{b}}(95 \% \mathrm{Cl})$ & \multicolumn{2}{|c|}{$3.82^{* * *}(2.79 \sim 5.23)$} & \multicolumn{2}{|l|}{1.00} & \multicolumn{2}{|c|}{$3.47^{* * *}(2.26 \sim 5.31)$} & \multicolumn{2}{|c|}{1.00} \\
\hline
\end{tabular}

Notes: 'Stratified Cox proportional regression stratified on patients' age group and index year

${ }^{\mathrm{b}}$ Adjusted for patients' monthly income, urbanization level, geographic region, hypertension, diabetes, obesity, tobacco use disorder, and alcohol abuse/alcohol dependence syndrome. ${ }^{* *} p<0.001$

suggest that persistent activation of the hypothalamicpituitary-adrenal (HPA) axis can alter the function of the neuroendocrine system, disrupt the circadian glucocorticoid rhythm, and thus promote tumor initiation and progression [28-31]. More specifically, stress-related neurotransmitters such as serotonin were proposed as being correlated with the progression of urological cancers $[32,33]$.

As an important monoamine neurotransmitter in the brain, serotonin plays a crucial role in the neurobiological processing of anxiety. Dysregulation of serotonin is strongly associated with the development of $\mathrm{ADs}$ [34]. Serotonin also serves as a messenger that controls several processes of the body including carcinogenesis. Siddiqui and colleague found that serotonin could increase the proliferation of bladder cancer cells in vitro in a dose-dependent manner and could modify the growth inhibitory effect of doxazosin on prostate and bladder cancer cells $[15,35]$. Based on relevant findings, serotonin was proposed to be a prognostic marker for urological cancer [36]. Although the majority of previous studies were conducted in vitro and clinical applications remain controversial [37]. We argue that dysregulation of the serotonin receptor pathway might contribute to the relationship between $\mathrm{ADs}$ and urological cancers.

Lines of evidence support that traumatic experience may alter core physiological systems and even cause carcinogenesis through DNA breakage [38]. Traumatic experience, even early childhood maltreatment, may increase the risk of ADs [39]. On the other hand, effective treatment such as psychotherapy could reverse DNA damage [40]. There could be a remote and common cause for both ADs and urological cancers. The strength of this study comes from it utilizing a large, population-based sample. Still, the results should be interpreted in light of potential methodological limitations. First of all, the use of non-standardized diagnoses made by different physicians is an inherent problem with population-based studies. By restricting our analyses to two visits with a diagnosis of
ADs with at least one having been made by a boardcertified psychiatrist, we enhanced the specificity of the psychiatric diagnoses. Further, we chose a broad approach to a diagnosis of ADs in order to reduce misclassification. Second, information about certain risk factors for urological cancer including environmental exposure, amount of tobacco and alcohol use, body mass index and family cancer history is not available in the database. Information on traumatic events and critical life events is also unavailable. We adjusted for the geographical location and urbanization level of each subject's residence in the study. The diagnoses of tobacco and alcohol use disorders, which represent the most severe form of tobacco and alcohol use, were also considered in our analyses. In addition, the LHID2005 provides no information about serotonin levels or the serotonin axis. Consequently, we could not demonstrate the notion whether serotonin is important in patients with ADs developing bladder cancer. Still, a potential bias could exist. And last, a 5-year follow up period was chosen to ensure an adequate time window for the development of urological cancers among subjects at risk. Some subjects might have had a more-insidious onset and could not be identified within the 5-year follow-up period.

\section{Conclusions}

Despite these limitations, we found that during the 5-year follow-up period, the risk of developing urological caner was much higher among patients with ADs, compared to their counterparts without ADs, and this association was totally independent from comorbid hypertension, diabetes, obesity, and registered alcohol and tobacco use disorders. Clinicians should be aware of the increased risk of developing urological cancer among patients with ADs and are encouraged not to neglect relevant urological complaints.

\section{Abbreviations}

ADs: Anxiety disorders; HR: hazard ratio; LHID2005: Longitudinal health insurance database 2005; NHI: National health insurance; SIR: Standardized incidence ratio. 


\section{Acknowledgements}

This study is based in part on data from the National Health Insurance Research Database provided by the Bureau of National Health Insurance, Department of Health, Taiwan and managed by the National Health Research Institutes. The interpretations and conclusions contained herein do not represent those of the Bureau of National Health Insurance, Department of Health, or the National Health Research Institutes.

\section{Funding}

None.

\section{Availability of data and materials}

The LHID2005, which was open to the researchers in Taiwan, was available from the National Health Insurance Institute (http://law.moj.gov.tw/ LawClass/LawAll.aspx?PCode=10050021). Only citizens of Taiwan who fulfill the requirements of conducting research projects are eligible to apply for the LHID2005. The use of LHID2005 is limited to research purposes only.

\section{Authors' contributions}

YC participated in the design of the study and helped to draft the manuscript. HCLin, HCLee and LT performed the statistical analysis and helped to draft the manuscript. SD conceived of the study, participated in its design and coordination and helped to draft the manuscript. CC helped to revise the manuscript critically for important intellectual content and made substantial contributions to analysis and interpretation of data. All authors reviewed the manuscript.

\section{Competing interests}

The authors declare that they have no competing interests.

\section{Consent for publication}

Not applicable

\section{Ethics approval and consent to participate}

This study was exempt from full review by the Institutional Review Board of National Defense Medical Center since the LHID2005 consists of de-identified secondary data released to the public for research purposes.

\section{Author details}

'Department of Psychiatry, Shuang Ho Hospital, Taipei Medical University, New Taipei City, Taiwan. ${ }^{2}$ Graduate Institute of Life Science, National Defense Medical Center, Taipei, Taiwan. ${ }^{3}$ Sleep Research Center, Taipei Medical University, Taipei, Taiwan. ${ }^{4}$ Department of Psychiatry and Medical Humanities, School of Medicine, College of Medicine, Taipei Medical University, Taipei, Taiwan. ${ }^{5}$ School of Health Care Administration, Taipei Medical University, Taipei, Taiwan. ${ }^{6}$ Division of Urology, Department of Surgery, Far Eastern Memorial Hospital, New Taipei City, Taiwan. ${ }^{7}$ Graduate Program in Biomedical Informatics, College of Informatics, Yuan-Ze University, Chung-Li, Taiwan. ${ }^{8}$ Department of Surgery, Far Eastern Memorial Hospital, No.21, Sec. 2, Nanya S. Rd., Banciao Dist, New Taipei City 220, Taiwan.

\section{Received: 24 August 2016 Accepted: 7 November 2016}

Published online: 16 November 2016

\section{References}

1. Somers JM, Goldner EM, Waraich P, Hsu L. Prevalence and incidence studies of anxiety disorders: a systematic review of the literature. Can J Psychiatry. 2006;51:100-13.

2. Marques L, Robinaugh DJ, LeBlanc NJ, Hinton D. Cross-cultural variations in the prevalence and presentation of anxiety disorders. Expert Rev Neurother. 2011;11:313-22

3. Faravelli C, Guerrini Degl'Innocenti B, Giardinelli L. Epidemiology of anxiety disorders in Florence. Acta Psychiatr Scand. 1989;79:308-12.

4. Chen CN, Wong J, Lee N, Chan-Ho MW, Lau JT, Fung M. The Shatin community mental health survey in Hong Kong. II. Major findings. Arch Gen Psychiatry. 1993;50:125-33.

5. Mendlowicz MV, Stein MB. Quality of life in individuals with anxiety disorders. Am J Psychiatry. 2000;157:669-82.
6. Marciniak MD, Lage MJ, Dunayevich E, Russell JM, Bowman L, et al. The cost of treating anxiety: the medical and demographic correlates that impact total medical costs. Depress Anxiety. 2005;21:178-84.

7. Katon W, Lin EH, Kroenke K. The association of depression and anxiety with medical symptom burden in patients with chronic medical illness. Gen Hosp Psychiatry. 2007:29:147-55.

8. Chida Y, Hamer M, Wardle J, Steptoe A. Do stress-related psychosocial factors contribute to cancer incidence and survival? Nat Clin Pract Oncol. 2008;5:466-75.

9. Hamer M, Chida Y, Molloy GJ. Psychological distress and cancer mortality. J Psychosom Res. 2009;66:255-8.

10. Aro AR, De Koning HJ, Schreck M, Henriksson M, Anttila A, et al. Psychological risk factors of incidence of breast cancer: a prospective cohort study in Finland. Psychol Med. 2005;35:1515-21.

11. Goldacre MJ, Wotton CJ, Yeates D, Seagroatt V, Flint J. Cancer in people with depression or anxiety: record-linkage study. Soc Psychiatry Psychiatr Epidemiol. 2007:42:683-9.

12. Liang JA, Sun LM, Su KP, Chang SN, Sung FC, et al. A nationwide population-based cohort study: will anxiety disorders increase subsequent cancer risk? PLoS One. 2012;7:e36370.

13. Shen CC, Hu YW, Hu LY, Hung MH, Su TP, et al. The risk of cancer in patients with generalized anxiety disorder: a nationwide population-based study. PLoS One. 2013;8:e57399.

14. Siddiqui EJ, Shabbir M, Thompson CS, Mumtaz FH, Mikhailidis DP. Growth inhibitory effect of doxazosin on prostate and bladder cancer cells. Is the serotonin receptor pathway involved? Anticancer Res. 2005:25:4281-6.

15. Siddiqui EJ, Shabbir MA, Mikhailidis DP, Mumtaz FH, Thompson CS. The effect of serotonin and serotonin antagonists on bladder cancer cell proliferation. BJU Int. 2006:97:634-9.

16. Chen YH, Yeh HY, Wu JC, Haschler I, Chen TJ, Wetter T. Taiwan's National Health Insurance Research Database: administrative health care database as study object in bibliometrics. Scientometrics. 2011;86:365-80.

17. Jones ME, Swerdlow AJ. Bias in the standardized mortality ratio when using general population rates to estimate expected number of deaths. Am J Epidemiol. 1998;148:1012-7.

18. McLaughlin TP, Khandker RK, Kruzikas DT, Tummala R. Overlap of anxiety and depression in a managed care population: Prevalence and association with resource utilization. J Clin Psychiatry. 2006;67:1187-93.

19. Chung SD, Lin HC. Association between chronic prostatitis/chronic pelvic pain syndrome and anxiety disorder: a population-based study. PLoS One. 2013;8:e64630

20. Chung KH, Liu SP, Lin HC, Chung SD. Bladder pain syndrome/interstitial cystitis is associated with anxiety disorder. Neurourol Urodyn. 2014;33:101-5.

21. Lykouras L, Michopoulos J. Anxiety disorders and obesity. Psychiatrike. 2011; 22:307-13.

22. Sabourin BC, Hilchey CA, Lefaivre MJ, Watt MC, Stewart SH. Why do they exercise less? Barriers to exercise in high-anxiety-sensitive women. Cogn Behav Ther. 2011:40:206-15.

23. Sommer F, Klotz T, Schmitz-Drager BJ. Lifestyle issues and genitourinary tumours. World J Urol. 2004:21:402-13.

24. Kao CH, Sun LM, Su KP, Chang SN, Sung FC, et al. Benzodiazepine use possibly increases cancer risk: a population-based retrospective cohort study in Taiwan. J Clin Psychiatry. 2012;73:e555-60

25. Pottegard A, Friis S, Andersen M, Hallas J. Use of benzodiazepines or benzodiazepine related drugs and the risk of cancer: a population-based case-control study. Br J Clin Pharmacol. 2013;75:1356-64.

26. Selaman Z, Bolton JM, Oswald T, Sareen J. Association of benzodiazepine use with increased cancer risk is misleading due to lack of theoretical rationale and presence of many confounding factors. J Clin Psychiatry. 2012;73:1264.

27. Cohen S, Herbert TB. Health psychology: psychological factors and physical disease from the perspective of human psychoneuroimmunology. Annu Rev Psychol. 1996:47:113-42.

28. Sephton S, Spiegel D. Circadian disruption in cancer: a neuroendocrineimmune pathway from stress to disease? Brain Behav Immun. 2003;17:321-8.

29. Reiche EM, Nunes SO, Morimoto HK. Stress, depression, the immune system, and cancer. Lancet Oncol. 2004:5:617-25.

30. Antoni MH, Lutgendorf SK, Cole SW, Dhabhar FS, Sephton SE, et al. The influence of bio-behavioural factors on tumour biology: pathways and mechanisms. Nat Rev Cancer. 2006;6:240-8. 
31. Eismann EA, Lush E, Sephton SE. Circadian effects in cancer-relevant psychoneuroendocrine and immune pathways. Psychoneuroendocrinology. 2010;35:963-76.

32. Dizeyi N, Bjartell A, Nilsson E, Hansson J, Gadaleanu V, et al. Expression of serotonin receptors and role of serotonin in human prostate cancer tissue and cell lines. Prostate. 2004;59:328-36.

33. Shinka T, Onodera D, Tanaka T, Shoji N, Miyazaki T, et al. Serotonin synthesis and metabolism-related molecules in a human prostate cancer cell line. Oncol Lett. 2011;2:211-5.

34. Akimova $\mathrm{E}$, Lanzenberger R, Kasper $\mathrm{S}$. The serotonin-1A receptor in anxiety disorders. Biol Psychiatry. 2009;66:627-35.

35. Siddiqui EJ, Thompson CS, Mikhailidis DP, Mumtaz FH. The role of serotonin in tumour growth (review). Oncol Rep. 2005;14:1593-7.

36. Jungwirth N, Haeberle L, Schrott KM, Wullich B, Krause FS. Serotonin used as prognostic marker of urological tumors. World J Urol. 2008;26:499-504.

37. Ronkainen H, Soini Y, Vaarala MH, Kauppila S, Hirvikoski P. Evaluation of neuroendocrine markers in renal cell carcinoma. Diagn Pathol. 2010;5:28.

38. Schury K, Kolassa IT. Biological memory of childhood maltreatment: current knowledge and recommendations for future research. Ann N Y Acad Sci. 2012;1262:93-100

39. Green JG, McLaughlin KA, Berglund PA, Gruber MJ, Sampson NA, Zaslavsky AM, et al. Childhood adversities and adult psychiatric disorders in the national comorbidity survey replication I: associations with first onset of DSM-IV disorders. Arch Gen Psychiat. 2010;67(2):113-23.

40. Morath J, Moreno-Villanueva M, Hamuni G, Kolassa S, Ruf-Leuschner M, Schauer $\mathrm{M}$, et al. Effects of psychotherapy on DNA strand break accumulation originating from traumatic stress. Psychother Psychosom. 2014:83(5):289-97.

\section{Submit your next manuscript to BioMed Central and we will help you at every step:}

- We accept pre-submission inquiries

- Our selector tool helps you to find the most relevant journal

- We provide round the clock customer support

- Convenient online submission

- Thorough peer review

- Inclusion in PubMed and all major indexing services

- Maximum visibility for your research

Submit your manuscript at www.biomedcentral.com/submit

) Biomed Central 